\title{
Anti-inflammatory effects of total alkaloids from Rubus aleaefolius Poir. on non-alcoholic fatty liver disease through regulation of the NF-kB pathway
}

\author{
JINYAN ZHAO ${ }^{1}$, HAIYIN ZHENG ${ }^{2}$, YAN LIU ${ }^{2}$, JIUMAO LIN ${ }^{1}$, XIAOYONG ZHONG ${ }^{2}$, \\ WEI XU ${ }^{3}, Z^{2} H E N F E N G ~ H O N G{ }^{1}$ and JUN PENG ${ }^{1}$
}

\begin{abstract}
${ }^{1}$ Fujian Academy of Integrative Medicine, Biomedical Research Center, Fujian University of Traditional
Chinese Medicine, Fuzhou, Fujian 350122; Departments of ${ }^{2}$ Integrative Medicine, ${ }^{3}$ Pharmacy,

Fujian University of Traditional Chinese Medicine, Fuzhou, Fujian 350122, P.R. China
\end{abstract}

Received November 25, 2012; Accepted January 24, 2013

DOI: $10.3892 / \mathrm{ijmm} .2013 .1281$

\begin{abstract}
We aimed to explore the anti-inflammatory effects of total alkaloids in Rubus aleaefolius Poir. (TARAP) on nonalcoholic fatty liver disease, and to investigate the possible molecular mechanisms. A rodent non-alcoholic fatty liver disease (NAFLD) model was established by administration of a modified high-fat diet ad libitum for 8 weeks. Rats were treated with polyene phosphatidylcholine (PP), TARAP low-dose $(0.72 \mathrm{~g} / \mathrm{kg}$ body weight/day) and TARAP high-dose $(1.44 \mathrm{~g} / \mathrm{kg}$ body weight/day). The model group and the control group received distilled water. After treatment for 4 weeks, the blood samples were obtained from the abdominal aorta, and the levels of serum ALT, AST, GGT, ALP, TG, TC, HDL-C and LDL-C were measured. Changes in liver tissue morphology were evaluated by H\&E staining. The expression levels of nuclear factor (NF)- $\kappa \mathrm{B}$, cyclooxygenase-2 (COX-2), interleukin (IL)- 6 and tumor necrosis factor (TNF)- $\alpha$ in rat livers were assayed by reverse transcription-polymerase chain reaction (RT-PCR) and immunohistochemistry. Both TARAP and PP attenuated hepatic steatosis induced by the high-fat diet. The modified high-fat diet caused a significant increase in ALT, AST, GGT, ALP, TG, TC, LDL-C levels and a decrease in HDL-C levels. TARAP and PP treatment abrogated the increase in the levels of liver enzymes and the levels of TG, TC, LDL-C, as well as suppressed the increase in HDL-C levels. The results of RT-PCR and immunohistochemical assay showed that PP and TARAP treatment decreased the
\end{abstract}

Correspondence to: Dr Zhenfeng Hong or Dr Jun Peng, Fujian Academy of Integrative Medicine, Biomedical Research Center, Fujian University of Traditional Chinese Medicine, Fuzhou, Fujian 350122, P.R. China

E-mail: zfhong1953@163.com

E-mail: pjunlab@hotmail.com

Key words: total alkaloids, Rubus aleaefolius Poir., nonalcoholic fatty liver disease, nuclear factor- $\mathrm{kB}$ expression of NF- $\kappa \mathrm{B}, \mathrm{COX}-2$, IL- 6 and TNF- $\alpha$. In conclusion, these results suggest that TARAP may protect against NAFLD through regulation of the $\mathrm{NF}-\kappa \mathrm{B}$ pathway.

\section{Introduction}

Non-alcoholic fatty liver disease (NAFLD) is increasingly diagnosed worldwide and is considered to be the most common liver disorder in Western countries (1). It comprises a disease spectrum which includes variable degrees of simple steatosis (fatty liver), non-alcoholic steatohepatitis (NASH) and cirrhosis. Simple steatosis is benign, whereas steatohepatitis (NASH) is characterized by hepatocyte injury, inflammation and fibrosis (2), which can lead to cirrhosis, liver failure and hepatocellular carcinoma (3-6).

NAFLD affecting $10-24 \%$ of the general population in various countries (7-9) is strongly associated with obesity, type 2 diabetes (10), insulin resistance, hypertension and dyslipidemia (11), and is now regarded as the liver manifestation of the metabolic syndrome. The pathogenesis of non-alcoholic steatohepatitis is not fully understood, but the working hypothesis suggests that a first hit leads to the development of steatosis and is followed by a second hit that causes inflammation, ballooning and fibrosis (steatohepatitis) (12).

Nuclear factor (NF) $-\kappa B$, a nuclear transcription factor, regulates the expression of various genes, including interleukin (IL)-1 $\beta$, tumor necrosis factor (TNF)- $\alpha$, inducible NO synthase (iNOS), and cyclooxygenase-2 (COX-2), that play critical roles in apoptosis, tumorigenesis, various autoimmune diseases and inflammation $(13,14)$. Due to the ubiquitous role in the pathogenesis of inflammatory gene expression, $N F-\kappa B$ is a current target for treating various diseases (15). NF- $\kappa \mathrm{B}$ is activated in response to various inflammatory stimuli, including bacterial LPS, cytokines and viral proteins (16). Under normal conditions, $\mathrm{NF}-\kappa \mathrm{B}$ is present in the cytoplasm as an inactive heterotrimer consisting of p50, p65 and $\mathrm{I} \kappa \mathrm{B} \alpha$ subunits. Upon activation, degradation of $\mathrm{I} \kappa \mathrm{B} \alpha$ exposes a localization signal on the p50/p65 heterodimer, leading to nuclear translocation and binding to a specific sequence in several promoters, which in turn results in gene transcription of proinflammatory genes. 
Rubus (Rubus L.), a major genus of the Rosaceae family, is widely distributed throughout the world. The anti-inflammatory effects of Rubus coreanus (17), the antioxidant activities $(18,19)$, the hypoglycemic activity $(20)$, the apoptotic activity of a crude extract of Rubus crataegifolius roots (21) and the antimicrobial activity of Rubus ulmifolius (22) have been reported. Rubus aleaefolius Poir. (R. aleaefolius) is one species within Rubus spread throughout India, Southeast Asia, the Philippines, Japan, Taiwan and mainland China, Guizhou, Hunan, Fujian, Guangdong, Guangxi and Yunnan and other places. Its root or leaves are used as medicine, while the related activity of the edible fruit, in the research literature is rare in journals. $R$. aleaefolius is generally used as a folk medicinal herb to treat various types of hepatitis in the Anxi County of Fujian Province, China. Preliminary investigations by our study group based on carbon tetrachloride $\left(\mathrm{CCl}_{4}\right)$-induced acute liver injury model in rat in vivo showed that the total alkaloids found in Rubus aleaefolius Poir. (TARAP) ameliorated adipose degeneration caused by $\mathrm{CCl}_{4}$ (23).

In the present study, we fed rats with a modified high-fat diet (mHFD), and investigated whether TARAP has anti-inflammatory effects on liver metabolism and steatosis in the mHFD rat model, and investigated the possible mechanisms.

\section{Materials and methods}

Preparation and content assay of TARAP. The preparation of TARAP was performed as previously described (24). The roots of $R$. aleaefolius were collected from Anxi of Fujian Province, China, identified and authenticated by experts in our University, and the alkaloids were extracted as follows. The herb powder $(1 \mathrm{~g})$ was extracted using $50 \mathrm{ml}$ chloroform:methanol:ammonia solution $(15: 4: 3)$ for $2 \mathrm{~h}$ in an ice bath, sonicated for $30 \mathrm{~min}$, brought to room temperature and filtered. The filtered solution was collected and dessicated. The resultant residue was dissolved by $2 \mathrm{ml}$ of $2 \%$ sulfuric acid solution and filtered. The filter paper and residue were re-washed with $2 \mathrm{ml}$ of $2 \%$ sulfuric acid solution and with buffer solution ( $\mathrm{pH}$ 3.6). The buffer was then added to make a final volume of $50 \mathrm{ml}$, and the solution was saved for future use. Acid dye colorimetry was used to measure the total alkaloid content. The total alkaloid content was $0.81 \mathrm{mg}$ of alkaloid per gram of initial herb powder.

Reagents. TRIzol kit, M-MLV first-strand cDNA synthesis kit and TaqDNA polymerase were obtained from Invitrogen (Carlsbad, CA, USA). Assay kits for alanine aminotransferase (ALT), aspartate transaminase (AST), $\gamma$-glutamyltransferase (GGT), alkaline phosphatase (ALP), triglyceride (TG), total cholesterol (TC), high-density lipoprotein cholesterol (HDL-C) and low-density lipoprotein cholesterol (LDL-C) activity were obtained from the Jiancheng Institute of Biotechnology (Nanjing, China). All of the other chemicals, unless otherwise stated, were obtained from Sigma Chemicals Co. (St. Louis, MO, USA).

Animal model and treatment protocols. Male 8-week-old Sprague-Dawley (SD) rats (Slike Co., Ltd., Shanghai, China), weighing 180-200 g, were housed five per cage in an environmentally controlled room at a temperature of $22 \pm 1^{\circ} \mathrm{C}$ and relative humidity $40-60 \%$. Air ventilation was carried out 12-18 times/h and a 12:12 h artificial light/dark cycle of 150-300 lux was maintained. Food and water was provided ad libitum. The animal studies were approved by the Fujian Institute of Traditional Chinese Medicine Animal Ethics Committee (Fuzhou, China). The experimental procedures were carried out in accordance with the Guidelines for Animal Experimentation of Fujian University of Traditional Chinese Medicine (Fuzhou, China).

For the establishment of the aminal model, 60 rats were randomly divided into 5 groups (12 rats/group). One group of rats was used for the control. The remaining rats were fed a modified high-fat diet (mHFD) ad libitum for 8 weeks. The mHFD recipe we used to conform to NAFLD models of SD rat: $87.3 \%$ of basal fodder, $10 \%$ of lard, $2 \%$ of cholesterol and $0.7 \%$ of swine bile salt. After an 8 -week feeding period, the rats fed with the mHFD were randomly divided into 4 groups: the model group (model), polyene phosphatidylcholine-treated group (PP), TARAP-treated groups (high- and low-dose) (12 rats/group). PP (76 mg/kg body weight/day), TARAP high-dose $(1.44 \mathrm{~g} / \mathrm{kg}$ body weight/day) and TARAP low-dose $(0.72 \mathrm{~g} / \mathrm{kg}$ body weight/day) were administered as previously described (24). The model group and the control group received distilled water. The body weights and food uptake were recorded weekly. All rats were sacrificed by decapitation after $4 \mathrm{~h}$ of food deprivation. Blood samples were collected for assays of ALT, AST, GGT, ALP, TG, TC, HDL-C and LDL-C. Livers were rapidly dissected. A part of each liver was cut and fixed in formaldehyde saline (4\%) solution for histological analysis; the rest was snap frozen in liquid nitrogen and stored at $-70^{\circ} \mathrm{C}$ until use.

Histological examination. The liver tissue was immediately fixed in $10 \%$ buffered formalin for pathological analysis. Formalin-fixed liver tissue was paraffin-embedded and then sections of 4-5 $\mathrm{mm}$ were prepared and subsequently stained with hematoxylin and eosin (H\&E). Histological evaluation was performed twice by a pathologist unaware of the treatments on two separate occasions. A semiquantitative scoring system was used to assess the severity of the hepatic steatosis and inflammatory cell infiltration in 10 microscopic fields. In brief, the following criteria were used for scoring hepatic steatosis: grade $0(-)$, no fat; grade $1(+)$, fatty hepatocytes occupying $33 \%$ of the hepatic parenchyma; grade $2(++)$, fatty hepatocytes occupying 33-66\% of the hepatic parenchyma; grade $3(+++)$, fatty hepatocytes occupying $>66 \%$ of the hepatic parenchyma.

Biochemical assays. Serum was separated by centrifugation at $4^{\circ} \mathrm{C}$ and analyzed immediately or stored at $-20^{\circ} \mathrm{C}$. Serum ALT, AST, GGT, ALP, TG, TC, HDL-C and LDL-C levels were determined by spectrophotometry.

Reverse transcription-polymerase chain reaction (RT-PCR) analysis. Total RNA was isolated from liver with TRIzol and reverse transcribed by a two-step method using the SuperScript First-Strand Synthesis system. Following RT, the product was stored at $20^{\circ} \mathrm{C}$. The cDNA product $(1 \mu \mathrm{l})$ was added to prepare the PCR reaction mixture containing $200 \mu \mathrm{M}$ of dNTPs, $1.5 \mathrm{mM} \mathrm{MgCl}_{2}$ and 1.25 units Platinum Taq 
polymerase (Invitrogen) and 6 pmol of gene-specific primer sets for COX-2 or NF- $\kappa$ B or TNF- $\alpha$ or IL-6 or GAPDH. In each case, amplification was performed with a thermal cycler (GeneAmp PCR System 9600; Applied Biosystems, Foster City, CA, USA), using the following cycling parameters: denaturing at $95^{\circ} \mathrm{C}$ for $5 \mathrm{~min}$; amplification cycle at $95^{\circ} \mathrm{C}$ for $30 \mathrm{sec}$, annealing temperature for $30 \mathrm{sec}$ and $72^{\circ} \mathrm{C}$ for $1 \mathrm{~min}$; and final extension at $72^{\circ} \mathrm{C}$ for $10 \mathrm{~min}$. The expression of hepatic genes COX-2 and NF- $\kappa$ B or TNF- $\alpha$ or IL- 6 were analyzed. Six to eight samples were chosen randomly from each group and the assay of every gene in each sample was replicated three times. The rat GAPDH gene was amplified as a loading control. The PCR products were separated by electrophoresis on a $1.5 \%$ agarose gel. The genes and their forward and reverse primers are listed as follows: COX-2 forward, 5'-AC ACT CTA TCA CTG GCA CCC-3' and reverse, 5'-GA AGG GAC ACC CCT TCA CAT-3' (580 bp); NF- $\kappa$ B forward, 5'-GCA TTC TGA CCT TGC CTA T-3' and reverse, 5'-ATC CTT CCC AAA CTC CAC C-3' (377 bp); GAPDH forward, 5'-AGA TCC ACA ACG GAT-3' and reverse, 5'-TCC CTC AAG ATT GTC AGC AA-3' (308 bp); TNF forward, 5'-CAG CAG ATG GGC TGT ACC TT-3' and reverse, 5'-AAG TAG ACC TGC CCG GAC TC-3' (301 bp); IL-6 forward, 5'-CAA CTC CAT CTG CCC TTC-3' and reverse, 5'-TGG TCT TGG TCC TTA GCC-3' (596 bp).

Immunohistochemical assay. The paraffin-embedded liver samples were sectioned. Sections were subjected to antigen retrieval and blocking of endogenous peroxidase activity. For immunohistochemical staining, slides were incubated with rabbit polyclonal antibodies against COX-2, TNF- $\alpha$, IL-6 or NF- $\kappa$ B (all in a 1:200 dilution; Santa Cruz Biotechnology, Inc.). After washing with PBS, slides were incubated with biotinylated secondary antibody followed by conjugated horseradish peroxidase (HRP)-labeled streptavidin (Dako), and then washed with PBS. The slides were then incubated with diaminobenzidine (DAB) as the chromogen, followed by counterstaining with diluted Harris hematoxylin (both from Sigma). After staining, 5 high-power fields (x400) were randomly selected in each slide, and the average proportion of positive cells in each field was counted using the true color multi-functional cell image analysis management system (Image-Pro Plus; Media Cybernetics, USA). To rule out any non-specific staining, PBS was used to replace the primary antibody as a negative control.

Statistical analyses. All data are the means of three determinations, and data were analyzed using the SPSS package for Windows (version 11.5; SPSS, Inc., Chicago, IL, USA). Statistical analysis of the data was performed with the Student's t-test and ANOVA. Differences of $\mathrm{P}<0.05$ were considered statistically significant.

\section{Results}

Health condition of the rats. To determine the potential toxicity and safety of TARAP to rats, the condition of the rats and the changes in body weight were assayed. As shown in Fig. 1, the rats fed the mHFD for 8 weeks gained significantly more weight than rats fed a normal diet. TARAP supplementa-

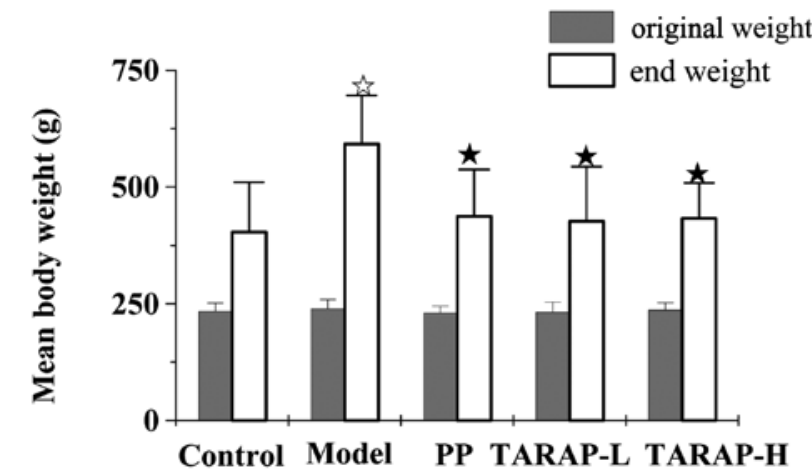

Figure 1. Effects of TARAP on body weight changes in SD rats fed the mHFD. The values represent means \pm SEM. ${ }^{2}<0.05$ (model vs. control), ${ }^{*} \mathrm{P}<0.05$ (high or low vs. model); ANOVA and post hoc test. TARAP-L, low-dose TARAP group; TARAP-H, high-dose TARAP group; PP, polyene phosphatidylcholine.

tion to the high-fat diet decreased the body weight. In addition, the body weight in the rats administered TARAP had no difference with the control group. These results showed that TARAP is safe for administration to SD rats.

Effect of TARAP on liver histology. Histological evaluation is regarded as the 'gold standard' approach with which to evaluate the presence and severity of NAFLD (25). Thus, we evaluated liver sections histologically to assess the extent to which TARAP attenuated the development of hepatic steatosis. Representative photomicrographs of the liver histology (Fig. 2) are shown. Rats fed a control diet had normal liver histology. However, rats fed the mHFD showed a high accumulation of fat and developed steatohepatitis, which was characterized by hepatocyte ballooning, scattered lobular inflammatory cell infiltration and inflammatory foci. Treatment with PP or TARAP clearly improved hepatic steatosis. Histological grading of liver sections confirmed that TARAP significantly ameliorated both hepatic steatosis and necroinflammation.

Effect of TARAP on the secretion of serum ALT, AST, GGT, $A L P, T G, T C, H D L-C$ and $L D L-C$. As shown in Fig. 3, the mHFD significantly increased serum ALT, AST, GGT, ALP, TC, TG and LDL-C levels $(\mathrm{P}<0.05)$ and reduced HDL-C levels $(\mathrm{P}>0.05)$ in the rats in the model group, when compared with the normal control group. However, the addition of PP or TARAP clearly neutralized the effect of the high-fat diet on the expression of these liver enzymes and blood lipids. The treatment of PP or TARAP reduced the levels of serum ALT, AST, GGT and ALP, whose levels were similar to the normal group. In addition, PP or TARAP treatment significantly suppressed the increased TC, TG and LDL-C levels induced by the mHFD $(\mathrm{P}<0.05)$, and upregulated the HDL-C levels, but without significance.

TARAP suppresses the expression of $N F-\kappa B, T N F-\alpha, C O X-2$ and IL-6 in the liver. The mRNA and protein expression of NF- $\kappa$ B, TNF- $\alpha$, COX- 2 and IL- 6 in the liver was determined by RT-PCR and immunohistochemical analysis, respectively. The results of the RT-PCR assay showed that PP or TARAP 


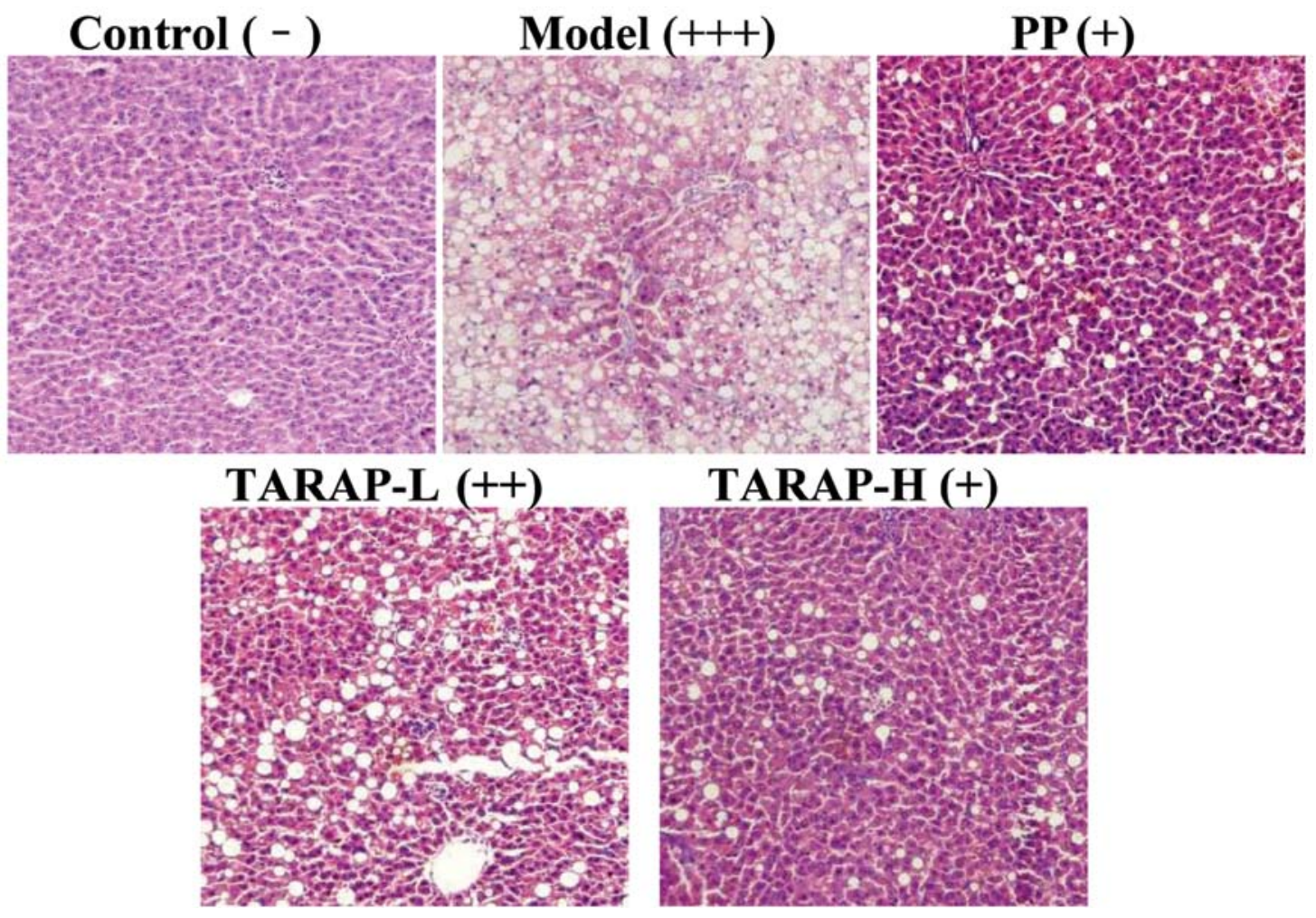

Figure 2. Effects of TARAP on the hepatic morphology of the liver tissues in rats fed the mHFD. Control (-), no liver fat; model (+++), fatty hepatocytes occupying $>66 \%$ of the hepatic parenchyma; $\mathrm{PP}(+)$, fatty hepatocytes occupying $33 \%$ of the hepatic parenchyma; TARAP-L (++), fatty hepatocytes occupying $33-66 \%$ of the hepatic parenchyma; TARAP-H (+), fatty hepatocytes occupying $33 \%$ of the hepatic parenchyma. Representative images were taken at a magnification, $x 400$. TARAP-L, low-dose TARAP group; TARAP-H, high-dose TARAP group; PP, polyene phosphatidylcholine.

treatment markedly reduced NF- $\kappa \mathrm{B}, \mathrm{TNF}-\alpha, \mathrm{COX}-2$ and IL-6 mRNA expression (Fig. 4). Data from the immunohistochemical assay showed that the protein expression patterns of NF- $\kappa B, T N F-\alpha, C O X-2$ and IL- 6 emulated the changes in the mRNA levels (Fig. 5). The percentages of cells positive for NF- $\kappa$ B, TNF- $\alpha$, COX- 2 or IL- 6 were significant in the model group $(89.88 \pm 8.09,89.68 \pm 9.31,67.2 \pm 2.02$ and $85.98 \pm 7.54 \%)$ when compared with the control group $(21.48 \pm 2.59,31.2 \pm 3.08$, $22.21 \pm 3.11$ and $22.15 \pm 3.67 \%$ ). Whereas these levels in the TARAP low- or high-treated rats were $57.8 \pm 5.69,50.31 \pm 5.66$, $27.92 \pm 2.65,59.38 \pm 4.21 \%$ and $27.19 \pm 3.39,42.12 \pm 3.87$, $31.67 \pm 2.58,47.09 \pm 5.55 \%$, respectively. Collectively, TARAP was found to attenuate inflammation induced by the mHFD via regulation of the NF- $\mathrm{kB}$ pathway.

\section{Discussion}

Non-alcoholic fatty liver disease (NAFLD) is one of the most common etiologies of chronic liver disease worldwide $(8,26)$. Although the pathogenesis of NAFLD remains undefined, various investigators have proposed a 'two hit' theory for the development of NASH. While the 'first hit' involves insulin resistance and results in the accumulation of fat in the liver, the 'second hit' includes oxidative stress resulting in lipid peroxidation, hepatocellular degeneration, cell death, hepatic stellate cell activation and fibrogenesis $(27,28)$. The two-hit hypothesis of steatohepatitis suggests that inflammation plays important roles in the development of steatohepatitis.
We succeeded in establishing an NAFLD rat model, This diet-induced model has more physiological similarities to human NASH than do models based on genetic defects or chemically induced disease. Moreover, cellular ballooning, one of the most important pathological features of NASH in humans, was observed in the mHFD model. This study was designed to investigate the possible beneficial effects of TARAP on NASH of rats fed an mHFD.

$R$. aleaefolius has been proven to have hepatoprotective activity on carbon tetrachloride-induced acute liver injury in rats in vivo. It can elevate the content of SOD and decrease TNF- $\alpha$ (29). These reports led us to hypothesize that TARAP may exert a beneficial effect on inflammation in NASH induced by a high-fat diet.

It is well known that cholesterol is a fatty substance which is an important part of the membrane of cells in the body of animals. The liver is capable of removing cholesterol from the blood circulation as well as manufacturing cholesterol and secreting cholesterol into the blood circulation. TC is a measure of the total amount of all cholesterol in blood at a given time and is the sum of HDL-C, LDL-C, very low-density lipoprotein cholesterol (VLDL-C), and intermediate density cholesterol (IDL). Triglycerides (TG) are the main lipids stored in the liver of NAFLD patients. Paradoxically, some experimental evidence shows that inhibition of TG synthesis improves liver steatosis but worsens liver damage, indicating that accumulation of liver TG may also be a protective mechanism to prevent liver damage in NAFLD (30). 

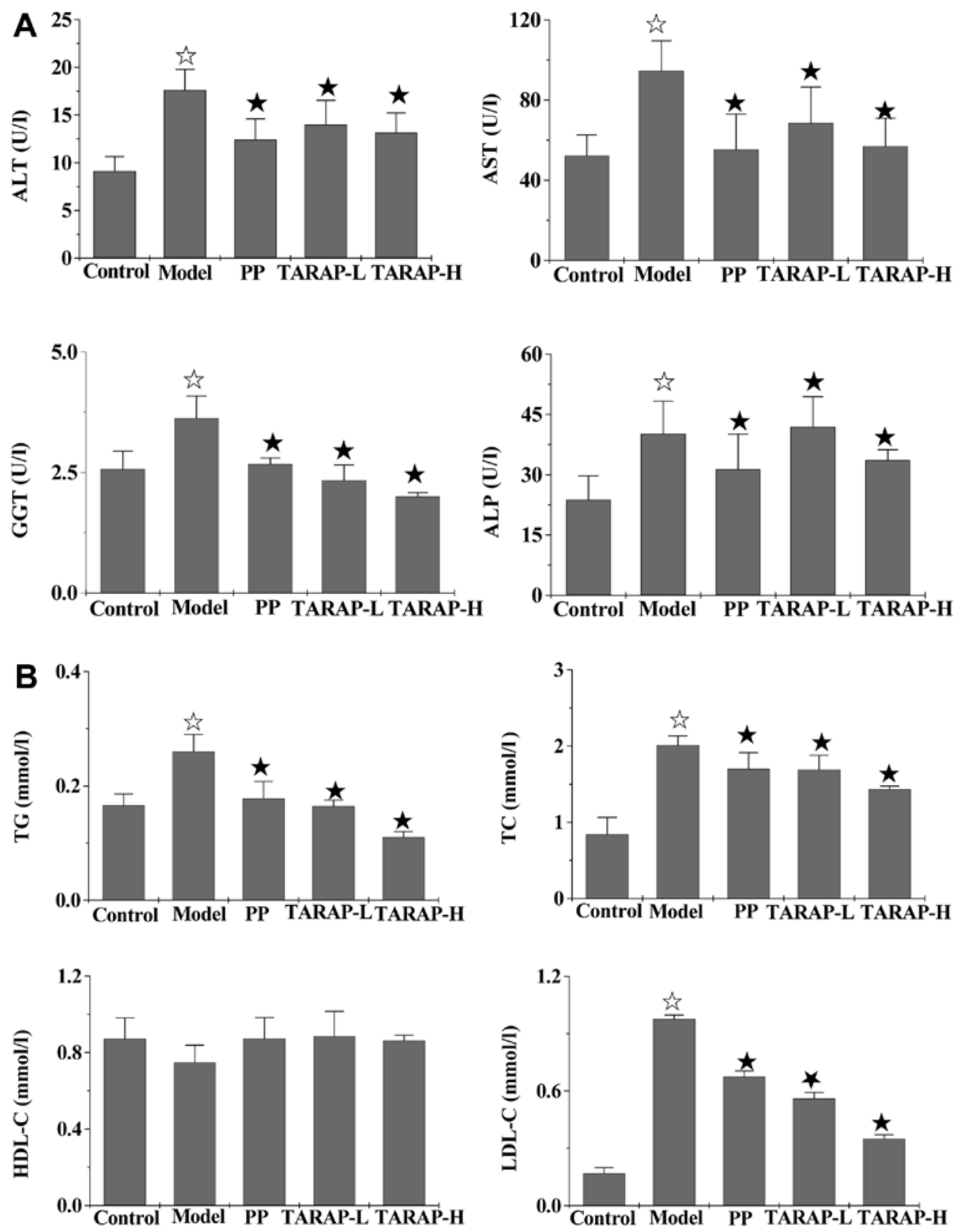

Figure 3. Effects of TARAP on mHFD-induced increases in hepatic enzymes and serum lipids in the rat groups. ${ }^{\text {t }}<<0.05$, compared with the control group; ${ }^{*} \mathrm{P}<0.05$, compared with the model group. PP, polyene phosphatidylcholine; TARAP-L, low-dose TARAP group; TARAP-H, high-dose TARAP group; ALT, alanine aminotransferase; AST, aspartate transaminase; GGT, $\gamma$-glutamyltransferase; ALP, alkaline phosphatase; TG, triglyceride; TC, total cholesterol; HDL-C, high-density lipoprotein cholesterol; LDL-C, low-density lipoprotein cholesterol.

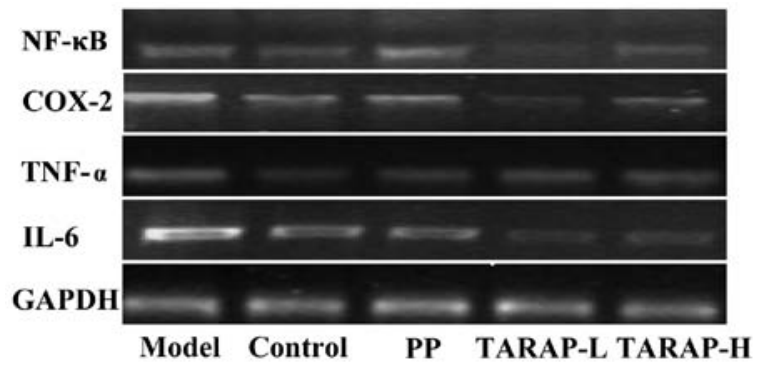

Figure 4. Effects of TARAP on NF- $\kappa$ B, COX-2, TNF- $\alpha$, IL-6 mRNA expression in mHFD-induced NAFLD rats. GAPDH was used as the internal control for the RT-PCR assay. This experiment was performed in triplicate and similar results were obtained. PP, polyene phosphatidylcholine; TARAP-L, low-dose TARAP group; TARAP-H, high-dose TARAP group.
As shown in Fig. 2B, the levels of TC and TG significantly increased in the model group. When mHFD-fed rats received TARAP, the levels of TC and TG evidently decreased. HDL-C removes excess cholesterol from arteries and moves it to the liver for further processing or to be eliminated from the body. A high serum HDL-C is beneficial. Therefore HDL-C is called 'good' cholesterol. LDL-C contributes to the build up of fat deposits in the arteries (atherosclerosis), which can cause decreased blood flow and heart attack. Thus, it is termed 'bad' cholesterol, and low levels are desirable. In the present study, we found high LDL-C levels and low HDL-C levels in the model rats, while PP and TARAP treatment significantly decreased the levels of LDL-C $(\mathrm{P}<0.05)$, and increased 

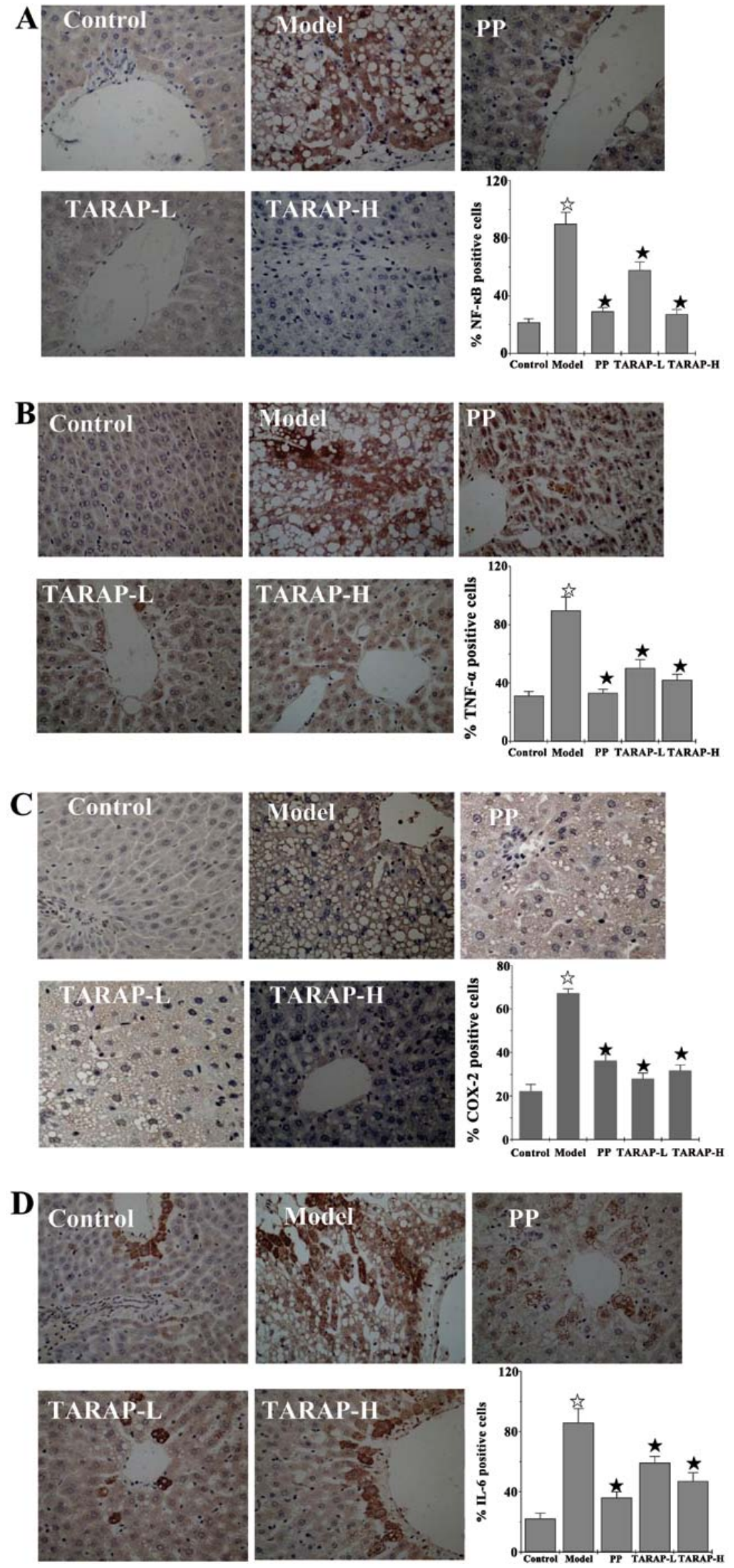

Figure 5. Protein expression and localization of hepatic (A) NF-кB, (B) TNF- $\alpha$, (C) COX-2 and (D) IL-6 in the control, model and the different experimental groups. Representative images were captured at a magnification, $\mathrm{x} 400$. Quantification of IHC assay is presented as a percentage of positively stained cells. Data shown are averages with $\mathrm{SD}$ (error bars) from 12 rats in each group. ${ }^{\text {* }} \mathrm{P}<0.05$, compared with the control group; ${ }^{*} \mathrm{P}<0.05$, vs. models. $\mathrm{PP}$, polyene phosphatidylcholine; TARAP-L, low-dose TARAP group; TARAP-H, high-dose TARAP group. 
HDL-C differentially, but without significance $(\mathrm{P}>0.05)$. The results reveal that TARAP modulates hyperlipidemia, and has protective effects against NASH.

The inflammatory response is a complex process involving many different signaling pathways. NF- $\mathrm{B}$ transcription factors regulate genes involved in many aspects of the inflammatory response (31). It has been shown that $\mathrm{NF}-\kappa \mathrm{B}$ activation is a key factor in the production of iNOS, COX-2 and various cytokines (TNF- $\alpha$, IL-6) in macrophages in response to LPS $(13,32)$. Since the expression of these proinflammatory mediators is modulated by $\mathrm{NF}-\kappa \mathrm{B}$, our findings suggest that treatment of rats fed an mHFD with TARAP effectively prevents $\mathrm{NF}-\kappa \mathrm{B}$ activation and the associated downregulation of several proinflammatory genes such as TNF- $\alpha$, IL- 6 and COX-2.

In summary, we demonstrated that TARAP treatment decreases the levels of ALT, AST, GGT, ALP, TC, TG, LDL-C in mHFD-fed rats. This anti-inflammatory effect occurs through the downregulation of COX-2, IL- 6 and TNF- $\alpha$ expression via the suppression of NF- $\mathrm{NB}$ activation. Thus, TARAP may be relevant for the clinical use for treatment of inflammatory diseases, including endotoxemia or sepsis.

\section{Acknowledgements}

The present study was supported by the Nature Science Foundation of the Fujian Province of China (no. 2010J01191 and 2010J01194) and the Project Sponsored by the Medical Originality Foundation of Fujian Province of China (no. 2009CX-18).

\section{References}

1. de Alwis NM and Day CP: Non-alcoholic fatty liver disease: the mist gradually clears. J Hepatol 48: S104-S112, 2008.

2. Angulo P, Keach JC, Batts KP and Lindor KD: Independent predictors of liver fibrosis in patients with nonalcoholic steatohepatitis. Hepatology 30: 1356-1362, 1999.

3. Matteoni CA, Younossi ZM, Gramlich T, et al: Non-alcoholic fatty liver disease: a spectrum of clinical and pathological severity. Gastroenterology 116: 1413-1419, 1999.

4. Teli MR, James OF, Burt AD, et al: The natural history of nonalcoholic fatty liver: a follow-up study. Hepatology 22: 1714-1719, 1995.

5. Day CP and James OFW: Steatohepatitis: a tale of two 'hits'? Gastroenterology 114: 842-845, 1998.

6. Cortez-Pinto $\mathrm{H}$, de Moura MC and Day CP: Non-alcoholic steatohepatitis: from cell biology to clinical practice. J Hepatol 44: 197-208, 2006.

7. Chen $\mathrm{CH}$, Huang $\mathrm{MH}$, Yang JC, et al: Prevalence and risk factors of nonalcoholic fatty liver disease in an adult population of Taiwan: metabolic significance of nonalcoholic fatty liver disease in nonobese adults. J Clin Gastroenterol 40: 745-752, 2006.

8. Bellentani S, Saccoccio G, Masutti F, et al: Prevalence of and risk factors for hepatic steatosis in Northern Italy. Ann Intern Med 132: 112-117, 2000.

9. Shen L, Fan JG, Shao Y, et al: Prevalence of nonalcoholic fatty liver among administrative officers in Shanghai: an epidemiological survey. World J Gastroenterol 9: 1106-1110, 2003.
10. Angulo P: Nonalcoholic fatty liver disease. N Engl J Med 346: 1221-1231, 2002.

11. Bugianesi E, McCullough AJ and Marchesini G: Insulin resistance: a metabolic pathway to chronic liver disease. Hepatology 42: 987-1000, 2005.

12. McCullough AJ: Update on non-alcoholic fatty liver disease. J Clin Gastroenterol 34: 255-262, 2002.

13. Lawrence T, Gilroy DW, Colville-Nash PR and Willoughby DA: Possible new role for $\mathrm{NF}-\kappa \mathrm{B}$ in the resolution of inflammation. Nat Med 7: 1291-1297, 2001.

14. Riehemann K, Behnke B and Schulze-Osthoff K: Plant extracts from stinging nettle (Urica dioica), an antirheumatic remedy, inhibit the proinflammatory transcription factor $\mathrm{NF}-\kappa \mathrm{B}$. FEBS Lett 442: 89-94, 1999.

15. Renard $\mathrm{P}$ and Raes $\mathrm{M}$ : The proinflammatory transcription factor NF-kappaB: a potential target for novel therapeutical strategies. Cell Biol Toxicol 15: 341-344, 1999.

16. Makarov SS: NF- $\kappa$ B as a therapeutic target in chronic inflammation: recent advances. Mol Med Today 6: 441-448, 2000.

17. Ywsilada E, Ustun O, Sezik E, et al: Inhibitory effect of Turkish folk remedies on inflammatory cytokines: interleukin-1 $\alpha$, interleukin- $1 \beta$ and tumor necrosis factor $\alpha$. J Ethnopharmacol 58: 59-73, 1997.

18. Costantino L, Albasini A, Rastelli G and Benvenuti S: Activity of polyphenolic crude extracts as scavengers of superoxide radicals and inhibitors of xanthine oxidase. Planta Med 58: 342-344, 1992.

19. Wang SY and Lin HS: Antioxidant activity in fruits and leaves of blackberry, raspberry, and strawberry varies with cultivar and development stage. J Agric Food Chem 48: 140-146, 2000.

20. Alonso R, Cadavid I and Calleja JM: A preliminary study of hypoglycemic activity of Rubus fruticosus. Planta Med 7 (Suppl): S102-S106, 1980.

21. Lee JH, Ham YA, Choi SH, et al: Activity of crude extract of Rubus crataegifolius roots as a potent apoptosis inducer and DNA topoisomerase I inhibitor. Arch Pharm Res 23: 338-343, 2000.

22. Rauha JP, Remes S, Heinonen M, et al: Antimicrobial effects of Finnish plant extracts containing flavonoids and other phenolic compounds. Int J Food Microbiol 56: 3-12, 2000.

23. Hong ZF, Li TJ and Zhao JY: Effect of total alkaloids of Rubus aleaefolius Poir on gene expressions of CYP2E2 and CYP3A1 in rats with acute liver injury. Zhongguo Zhong Xi Yi Jie He Za Zhi 29: 711-715, 2009 (In Chinese).

24. Lin JM, Zhao JY, Li TJ, et al: Hepatoprotection in a rat model of acute liver damage through inhibition of CY2E1 activity by total alkaloids extracted from Rubus alceifolius Poir. Int J Toxicol 30: 237-243, 2011.

25. Brunt EM: Nonalcoholic steatohepatitis: pathologic features and differential diagnosis. Semin Diagn Pathol 22: 330-338, 2005.

26. Bellentani S and Tiribelli C: The spectrum of liver disease in the general population: lesson from the Dionysos study. J Hepatol 35: 531-537, 2001.

27. Brunt EM: Pathology of nonalcoholic steatohepatitis. Hepatol Res 33: 68-71, 2005.

28. Farrell GC and Larter CZ: Nonalcoholic fatty liver disease: from steatosis to cirrhosis. Hepatology 43 (Suppl 1): S99-S112, 2006.

29. Ye HZ, Hong ZF, Wang YH and Chen YH: Effects of crude extract of Rubus Alcaefolius Poir on experimental liver injury in rats. Chin Arch Tradit Chin Med 23: 829-831, 2005.

30. Yamaguchi K, Yang L, McCall S, et al: Inhibiting triglyceride synthesis improves hepatic steatosis but exacerbates liver damage and fibrosis in obese mice with nonalcoholic steatohepatitis. Hepatology 45: 1366-1374, 2007.

31. Bonizzi G and Karin M: The two NF-kappaB activation pathways and their role in innate and adaptive immunity. Trends Immunol 25: 280-288, 2004.

32. Baldwin AS Jr: The NF-kappaB and I-kappaB proteins: new discoveries and insights. Annu Rev Immunol 14: 649-683, 1996. 